\title{
Performance Measurement of a New High-Resolution Detector System for I-131 Thyroid Studies
}

\author{
N. Bussmann, R. Engels, Member, IEEE, L. Fuss, G. Kemmerling, R. Reinartz, K.-J. Langen, J. Schelten, and \\ K. Ziemons
}

\begin{abstract}
A two-dimensional detector system for high-resolution thyroid I-131 scintigraphy was developed. It has a sensitive area of $4 \times 4 \mathrm{~cm}^{2}$ and consists of a lead collimator and an array of $10 \times 10 \mathrm{BGO}$ crystals combined with a position-sensitive photomultiplier. The spatial resolution and the sensitivity of the detector have been measured and compared to two commercially available gamma cameras. The full-width half-maximum for the new system is $3.8 \mathrm{~mm}$. Furthermore, first patient measurements have been carried out.
\end{abstract}

Index Terms-Detectors, I-131, scintigraphy, thyroid scanner.

\section{INTRODUCTION}

A T Forschungszentrum Juelich, a two-dimensional detector system was developed that is especially suited for studies of benign and malignant thyroid diseases during I-131 therapy [1]. It aims at a high spatial resolution to get precise information about I-131 distributions inside the thyroid. This was realized by using a collimator with an appropriate septa thickness and a lead matrix with $\mathrm{Bi}_{4} \mathrm{Ge}_{3} \mathrm{O}_{12}$ (BGO) crystals for the photon detection. Thus, the disadvantages [2] of conventional gamma cameras [3], [4] regarding spatial resolution could largely be avoided.

\section{DETECTOR AND COLLIMATOR}

The detector consists of a parallel hole lead collimator of variable thickness, which is placed in front of an array of $10 \times 10$ BGO crystals with a single size of $2 \times 2 \times 10 \mathrm{~mm}^{3}$ (Fig. 1). The BGO crystals were coupled to a position-sensitive photomultiplier tube (Hamamatsu R2487) for light detection. As Compton scattered events inside the BGO lead to crosstalks between adjacent cells and hence to a decrease of the spatial resolution, the crystals have been separated by $1.9-\mathrm{mm}$ lead surrounding, which was realized through a 10-mm-thick matrix. The resulting sensitive area of the detector is $4 \times 4 \mathrm{~cm}^{2}$. Also, the collimator has been tailored for a higher spatial resolution. The circular collimator holes have a diameter of $1.8 \mathrm{~mm}$ and are exactly positioned above the BGO crystals. Thus, there is a $2.2-\mathrm{mm}$ lead distance between adjacent holes to reduce septa penetration effects. Furthermore, the photomultiplier and the BGO matrix has been

Manuscript received October 13, 2000; revised March 12, 2001.

N. Bussmann was with Zentrallabor für Elektronik, Forschungszentrum Juelich GmbH, Juelich D-52425, Germany. She is now with Kretztechnik GmbH, D-45768 Marl, Germany (n.bussmann@kretz.co.at).

R. Engels, L. Fuss, G. Kemmerling, R. Reinartz, and K. Ziemons are with Zentrallabor für Elektronik, Forschungszentrum Juelich GmbH, Juelich D-52425, Germany (e-mail: r.engels@fz-juelich.de; 1.fuss@fz-juelich.de g.kemmerling@fz-juelich.de; r.reinartz@fz-juelich.de; k.ziemons@fz-juelich. de).

K.-J. Langen is with the Institut für Medizin, Forschungszentrum Juelich GmbH, Juelich D-52425, Germany (e-mail: k.j.langen@fz-juelich.de).

Publisher Item Identifier S 0018-9499(01)05269-8.

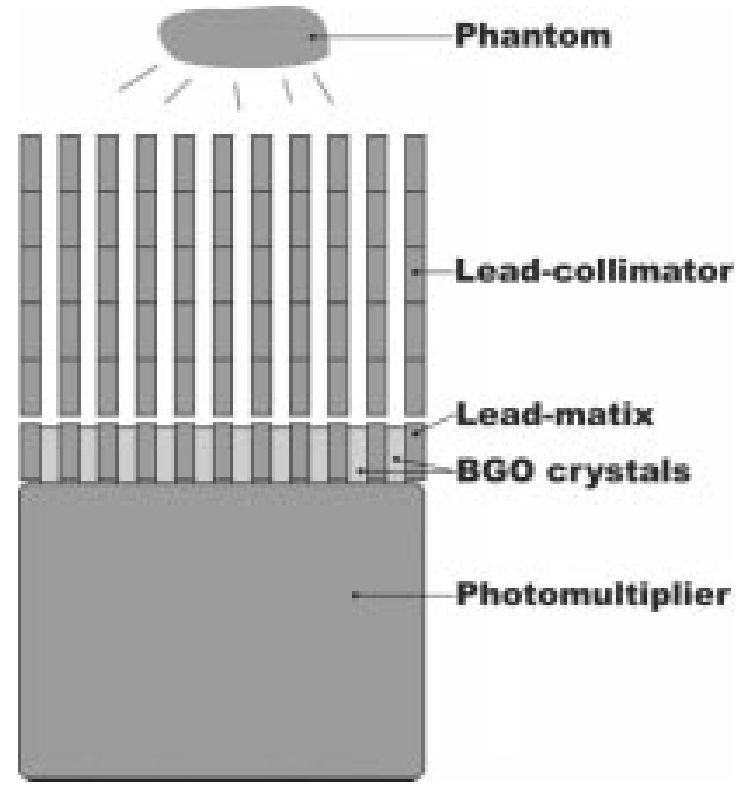

Fig. 1. Schematic drawing of the detector head.

surrounded by a 5 -mm-thick lead shielding in order to avoid the detection of lateral radiation from the subject under study.

Because of the relatively thick septa, there are dead areas on the acquired scintigramms. Therefore, the whole system is placed on a two-dimensional unit capable to position the system in the $x, y$-plane.

By moving the detector stepwise during measurements, several images are obtained, which are afterwards combined to achieve a final scintigramm without insensitive areas. Additionally, there is a height-adjustable table available to position patients in $z$-direction (Fig. 2). In order to calculate the position of the event, the signal ratios $Q_{x}$ and $Q_{y}$ have to be calculated in the field-programmable gate array according to (1) and (2).

\section{EXPERIMENTAL RESULTS}

The spatial resolution and the sensitivity of the detector have been measured with a collimator length of $60 \mathrm{~mm}$. To compare the results with commercial systems, also two available gamma cameras, a Picker-Dyna-4 and a single head of a Trionix-Triad, have been surveyed under identical measurement conditions.

The spatial resolution was determined according to the Nema standard with a 0.9-mm-wide line source, which has been filled with I-131 and positioned $100 \mathrm{~mm}$ above the collimator. The full-width half-maximum (FWHM) and full-width tenth-maximum (FWTM) were calculated from the measured line spread 


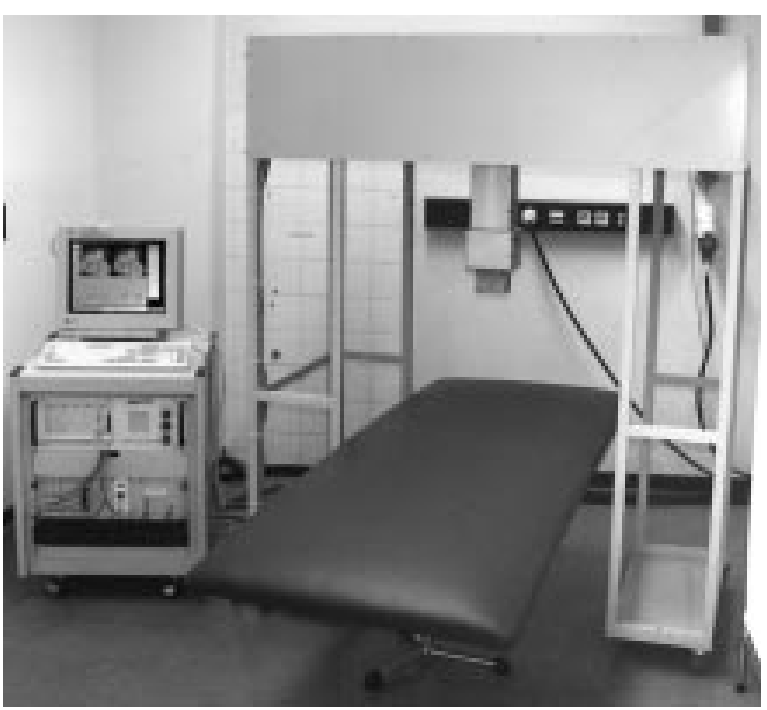

Fig. 2. Construction of the detector system.

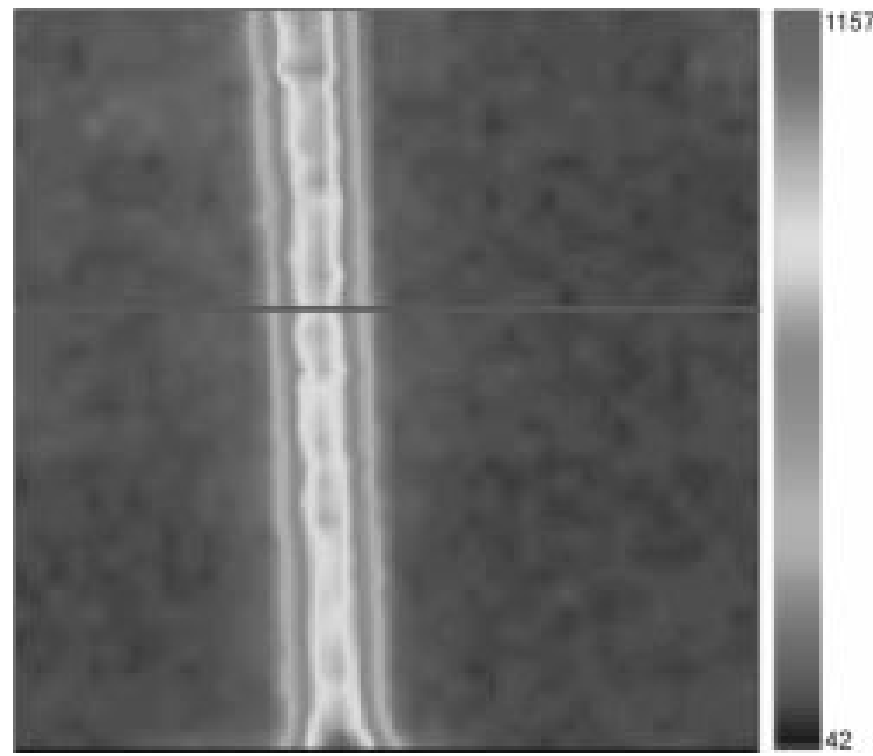

Fig. 3. Two-dimensional plot of a line source measured with the new detector.

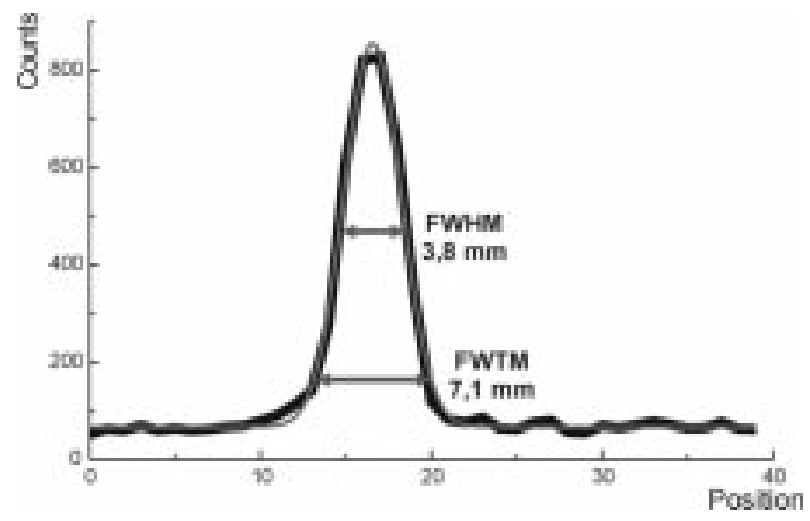

Fig. 4. Line scan of the two-dimensional plot.

function (Figs. 3 and 4). The results are listed in Table I, showing a significantly higher resolution for the developed detector.

This may also be demonstrated by two-dimensional measurements with a small thyroid phantom. The planar size of the thy-
TABLE I

SPATIAL RESOlution OF THE SySTEMS

\begin{tabular}{|c|c|c|}
\hline & FWHM & Fातन \\
\hline $\begin{array}{l}\text { Dereloped Detector } \\
\text { System }\end{array}$ & $38 \mathrm{~mm}$ & $7.1 \mathrm{~mm}$ \\
\hline Picker-Gamma-Camera & $84 \mathrm{minm}$ & $401 \mathrm{~mm}$ \\
\hline Troonix-Triad & 10.2mm & $201 \mathrm{~mm}$ \\
\hline
\end{tabular}

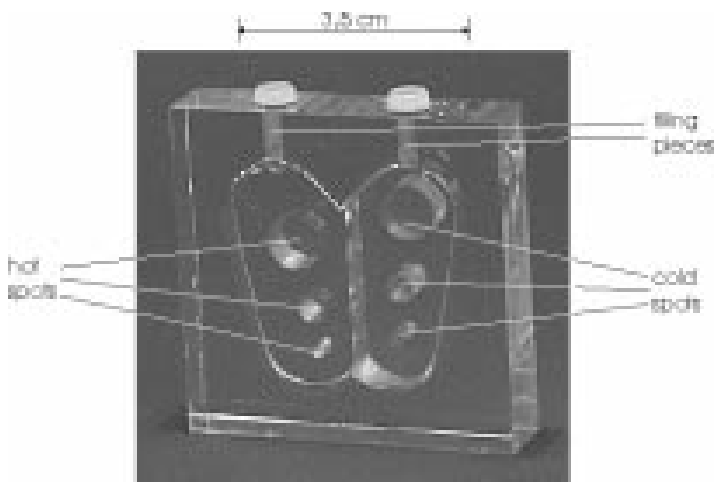

Fig. 5. Small thyroid phantom.

roid phantom is $35 \times 35 \mathrm{~mm}^{2}$. It consists of three hot and three cold spots of 2, 4, and $8 \mathrm{~mm}$ diameter (Fig. 5) and was filled with $73 \mathrm{MBq}$ I-131. In contrast to the commercial systems, the picture of the developed detector clearly shows all outlines and structures of the phantom (Figs. 6-8; acquisition time $32 \mathrm{~min}$ ).

For the determination of the sensitivity, a plane source of $100 \mathrm{~mm}$ diameter and $3 \mathrm{~mm}$ height was filled with $\mathrm{I}-131$ and positioned $100 \mathrm{~mm}$ above the collimator. While this experimental setup is equivalent to the Nema standard, for the determination of the sensitivity of the developed system, the Nema standard is not directly applicable. Nema requires the use of a plane source of $100 \mathrm{~mm}$ diameter, whereas the sensitive area of the developed detector is only $38 \times 38 \mathrm{~mm}$. Thus, the area of the phantom exceeds the sensitive area of the detector. In order to achieve a measure of the sensitivity, the ratio of the surface of the phantom and the surface of the sensitive area was calculated. The assigned value equal to 5.4 was multiplied with the determined counts per minute/MBq measured with the $100 \mathrm{~mm}$ diameter phantom positioned $100 \mathrm{~mm}$ above the collimator. A sensitivity of $\sim 500$ counts $/ \mathrm{min} / \mathrm{MBq}$ was calculated. The measured sensitivity of the Picker-Gamma camera with the $100 \mathrm{~mm}$ diameter phantom and a distance of $100 \mathrm{~mm}$ was 2386 counts $/ \mathrm{min} / \mathrm{MBq}$, and for the Trionix-Triad, a total sensitivity of 1172 counts/min/MBq was achieved. Here, the two commercial systems were superior to the developed detector by a factor of two to five. The relatively long and thick collimator septa, which favor a high resolution, affect prejudicially the sensitivity by large absorption of initial radiation. Nevertheless, also the achieved sensitivity allows for patient-friendly measurement times in the range of $15 \mathrm{~min}$.

\section{Patient Measurements}

In addition, first patient measurements have been carried out. Fig. 9 demonstrates the results of a patient with thyroid cancer 


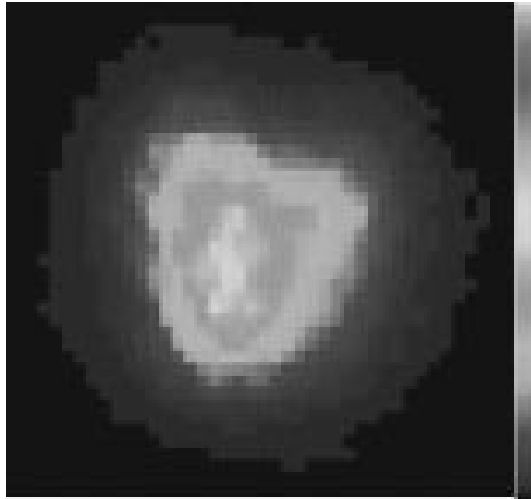

Fig. 6. Small thyroid phantom measured with the Trionix-Spect camera.

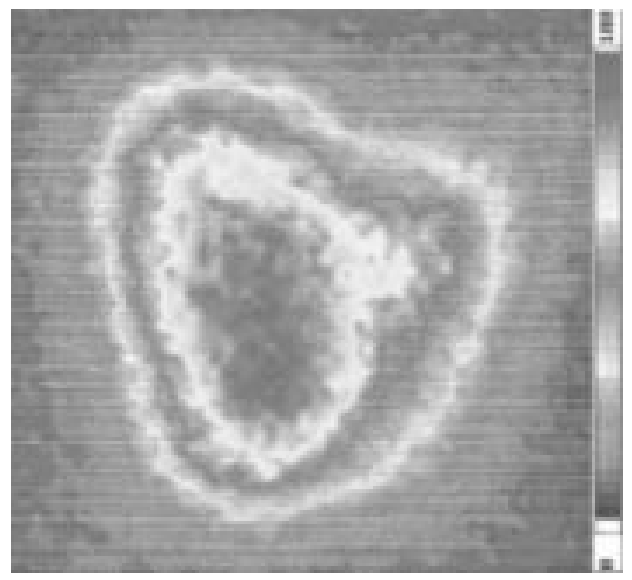

Fig. 7. Small thyroid phantom measured with the Picker-Gamma camera.

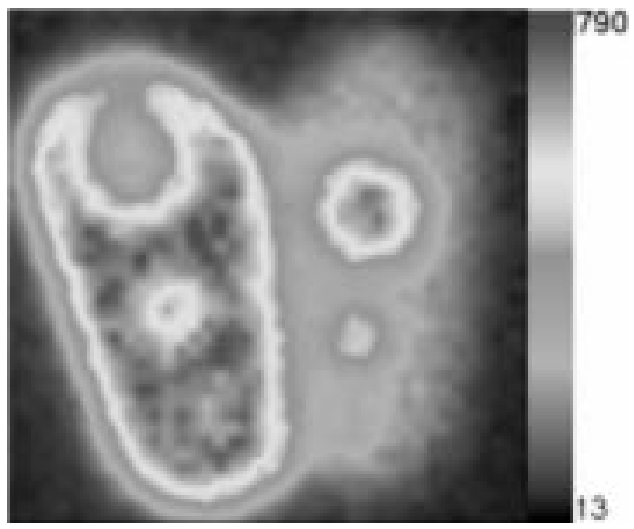

Fig. 8. Small thyroid phantom measured with the developed detector system.

$72 \mathrm{~h}$ after administration of $3.7 \mathrm{GBq}$ I-131 for ablation therapy. While the Picker-Gamma camera visualizes a large area with homogeneous I-131 uptake, the new system is able to identify several hot areas (acquisition time $16 \mathrm{~min}$ ). This adds significant information to the physician for patient management (Fig. 10).

\section{CONCLUSION}

Measurements of the spatial resolution and sensitivity of the developed detector system have been presented. Compared to commercial gamma cameras, the spatial resolution has been significantly improved, which was shown by measurements of line

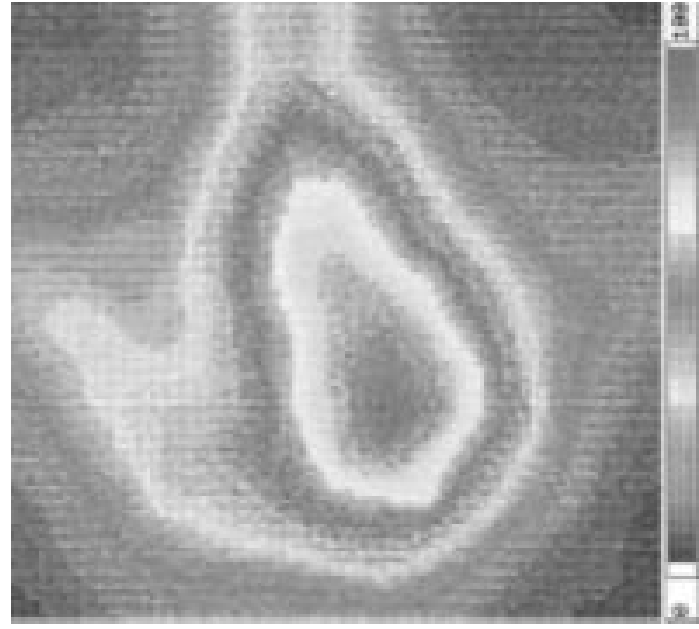

Fig. 9. Patient measurement with the Picker-Gamma camera.

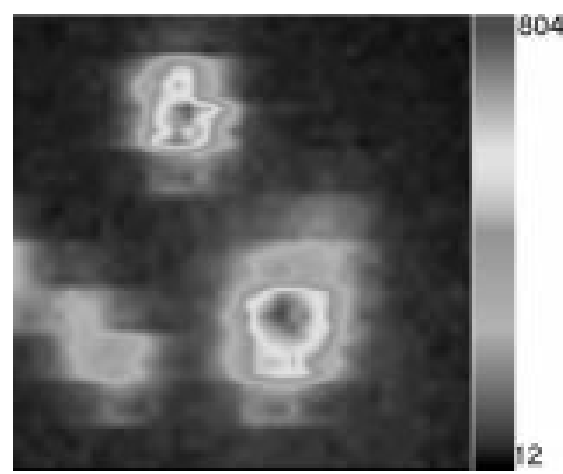

Fig. 10. Patient measurement with the developed detector system.

and thyroid phantoms. On the other hand, the achieved sensitivity is lower due to the length and thickness of the collimator septa but is still tolerable for patient-friendly measurements.

First results of patient measurements were promising, and a clinical study has been started to evaluate the impact of the method for patient management. Since the sensitive area of the presented system is rather small, a detector with a larger sensitive area using a Hamamatsu 5-in photomultiplier is under development.

\section{ACKNOWLEDGMENT}

The authors wish to thank Prof. Dr. C. Reiners, Dr. Lassmann, and Dr. Haenscheid, Klinik und Poliklinik fuer Nuklearmedizin der Universitaet Wuerzburg, for helpful scientific discussions and support.

\section{REFERENCES}

[1] N. Bussmann, F. Sonnenberg, R. Engels, R. Reinartz, N. Schramm, W. Friedrich, K.-J. Langen, and H. Halling, "Development of a high resolution, 2D detector system for iondine-131-scintigraphy," IEEE Trans. Nucl. Sci., vol. 47, pp. 1085-1087, June 2000.

[2] S. Hertz and A. Roberts, "Radioactive iodine as indicator in thyroid physiology: Use of radioactive iodine in differential diagnosis of 2 types of Graves' disease," J. Clin. Invest., vol. 21, no. 31, 1942.

[3] R. Wolf, Handbuch der medizinischen Radiologie. Nuklearmedizin: Springer-Verlag, 1980.

[4] J. C. W. Crawly and N. Veall, "The design and some clinical applications of a hybrid scanner," in Medical Radioisotope Scintigraphy, Vienna, Austria: IAEA, 1973, vol. 1, p. 105. 